\title{
A las puertas del cambio en la Formación Universitaria
}

\author{
On the verge of change in University Education
}

Ana María Escayola Maranges y Montserrat Vila Gangolells.

Escuela de Ciencias de la Salud de Manresa. Fundación Universitaria del Bages (FUB)

Los cambios sociales conducen a un nuevo planteamiento en la docencia universitaria. Es necesario un modelo educativo abierto para formar nuevos profesionales competentes, basado en la capacidad de aprender del alumno, de obtener información y de adaptarse a las situaciones de cambio que presenta la profesión. La planificación de programas educativos plantea nuevos roles en la función del docente y del alumno, exige cambios metodológicos en el proceso de aprendizaje, conllevando cambios en las estrategias educativas. La nuevas tecnologías de la información y comunicación son un elemento indispensable para promover el cambio, aunque no suficientes. El aprendizaje basado en problemas (ABP) ha resultado un elemento innovador en el ámbito universitario, demostrando un aumento de la motivación para aprender, potenciando el trabajo grupal, incentivando la búsqueda de información, aumentado la comprensión y la memorización del conocimiento. A su vez, el ABP pone énfasis en la interdisciplinariedad, aumenta el espíritu de colaboración del estudiante y su nivel de responsabilidad en el aprendizaje. En el ámbito de las Ciencias de la Salud, los métodos de aprendizaje son claves en el proceso formativo de profesionales competentes, dada la estrecha vinculación entre el ámbito laboral y formativo.

Palabras Clave: Educación, docencia, aprendizaje basado en problemas, competencia, espacio europeo de educación superior.

Correspondencia:

Ana María Escayola Maranges

Escuela de Ciencias de la Salud de Manresa.

Fundación Universitaria del Bages (FUB)

C/ Ramón d'Iglesias, 5-7

08242- Manresa

Fax: 938757355

Telf: 938774181

e-mail: aescayola@fub.edu
Changes in society as a whole oblige University education to introduce changes as well. In order to train well-equipped professionals, an open educational model is necessary, based on the ability of the student to learn, to obtain information, and to adapt to the changes inside the profession. The planning of learning programs involves new roles for the teacher and new functions for the student. It requires changes in methodology and in educational strategies. The new information and communication technologies are indispensable instruments for promoting these changes, but they are not enough in isolation. Problembased learning (PBL) is an innovative instrument in the University setting, increasing students' motivation, empowering group work, stimulating the search for information and increasing the comprehension and memorizing of knowledge. PBL also emphasizes interdisciplinary work, stressing the need to cooperate and to increase students' responsibility for their learning. In the field of health sciences, effective learning methods are vital for the training of competent professionals due to the close connection between the educational and work environments.

Key words: Education, teaching, problem based learning (PBL), competency, higher education in the European region.

\section{INTRODUCCIÓN}

Una de las funciones del sistema educativo es la de fomentar el aprendizaje, la cultura y los valores sociales de las nuevas generaciones mejorando el razonamiento crítico, las habilidades y actitudes de los estudiantes. Sin embargo los rápidos cambios 
que genera la sociedad actual obligan a un aprendizaje rápido y a una gran adaptabilidad por parte de los profesores. A su vez, al alumno se le exige una gran capacidad de asimilación, versatilidad, adaptación, y capacidad de innovación ${ }^{1}$.

Jacques Delors plantea cuatro pilares en la educación: aprender a conocer, a hacer, a convivir y a ser. González Seara añade a estos pilares la capacidad de aprender a pensar fomentando la innovación. Propone que la educación universitaria debería promover la generación de nuevas ideas con contenido científico y tecnológico que permitan su aplicación y ejecución en el ámbito profesional. Se debería plantear una educación pluridimensional que permitiera combinar la formación con el trabajo ${ }^{2}$.

Los nuevos sectores productivos exigen un perfil profesional altamente competente con un alto grado de experteza. Existen diferentes enfoques teóricos opuestos de cómo se consigue el nivel de experto profesional: el enfoque racionalista (formulado por reglas y normas) y el enfoque experiencial (basado en estilos libres de conducta). En enfermería, Dreyfus describe un modelo de cinco niveles diferentes para la adquisición de la experteza, remarcando la percepción y la toma de decisiones desde la perspectiva experiencial ${ }^{3}$. Para ello el ámbito docente ha cambiado el enfoque de la enseñanza superior "centrada en el profesor" por otra "centrada en el alumno" 4,5 donde el docente tiende a reemplazar su función de emisor de información por la de tutor del proceso de aprendizaje ${ }^{1}$. Se tiende a un nuevo modelo educativo abierto al mundo y a la vida, donde la educación se centra en la capacidad de aprender, en saber encontrar información y en la adaptación a situaciones nuevas y cambiantes ${ }^{2}$

La planificación de los programas educativos debe responder las siguientes cuestiones: a quién se enseña, quién aprende, cómo se debe enseñar/ aprender, y cómo controlar el aprendizaje. Los objetivos educativos no definen lo que el profesor es capaz de enseñar sino aquello que el estudiante debe aprender al término del proceso de enseñanza/aprendizaje. Una definición clara de los objetivos educativos llevará a una mayor rentabilidad de los medios pedagógicos, a una mejora en el aprendizaje y a una correcta evaluación del alumno. En este sentido, el trabajo del profesor es muy relevante para ayudar al alumno en su formación. Requiere de un cambio en cuanto a estrategias didácticas y procesos pedagógicos que respeten los objetivos educativos planteados en la Declaración de Bolonia ${ }^{5}$, en la Declaración mundial sobre la educación superior en el Siglo XXI y en el informe sobre la Universidad 2000 en España ${ }^{1,6,7}$.
Una de las principales contribuciones a este cambio es el avance y la aplicación de las Tecnologías de la Información y la Comunicación (TIC) en el campo educativo. Éstas abren un gran abanico de posibilidades en modalidades formativas que pueden situarse tanto en el ámbito de la educación a distancia, como en las modalidades de la enseñanza presencial. Salinas ${ }^{8}$ afirma que éstas pueden ser un punto de encuentro en el planteamiento del aprendizaje "abierto" propugnado por Lewis y Spencer ${ }^{9}$. Este sistema permite un alto grado de interacción y de control de la comunicación, en función de las capacidades y recursos tecnológicos, teniendo en cuenta las capacidades didácticas del docente. Por ello, existe un problema eminentemente pedagógico. Así, la tecnología no pretende dejar el aprendizaje en manos exclusivamente de los alumnos ni, por el contrario, la simple presencia del profesor predispone a una comunicación didáctica más directa y efectiva. De este modo, el concepto de "distancia" adquiere una significación de estudio sin ningún tipo de apoyo ${ }^{10}$, aunque esto puede suceder también en la enseñanza presencial. $\mathrm{Al}$ margen del concepto de enseñanza a distancia o enseñanza presencial, el proceso de aprendizaje abierto tiene en cuenta dos dimensiones ${ }^{11}$ :

1. Una relacionada con los determinantes administrativos del concepto "distancia" (lugar, tiempo, número de sesiones...)

2. Otra, relacionada con la traslación de los determinantes educacionales: metas de aprendizaje, secuencia y lugar de enseñanza, estrategia, etc. Aquí adquieren importancia los materiales didácticos.

Las instituciones que ofrecen formación presencial están utilizando las nuevas tecnologías como recurso didáctico y como herramienta para flexibilizar el entorno de enseñanza/aprendizaje. De ahí la posibilidad de programas mixtos que permitan la asistencia a pocas clases para seguir la formación desde sus casas, con la posibilidad de acudir a los profesores cuando sea necesario. Este sistema flexible permite la formación de numerosas personas con obligaciones familiares y laborales, pudiendo seguir la formación a lo largo de sus vidas.

Bajo esta perspectiva, la utilización de las TIC en la enseñanza flexible de la formación, obliga a plantearse diferentes frentes de renovación educativa ${ }^{8}$ : cambios en las concepciones de cómo funciona el aula (definición de los procesos didácticos, identidad del docente...), cambios en los recursos básicos (contenidos, infraestructuras, eficacia didáctica...) $\mathrm{y}$, finalmente, cambios en las prácticas (de los profesores y de los estudiantes) 
En este sentido, la implantación de nuevas estrategias centradas en el "aprendizaje basado en problemas" (ABP) ha resultado un elemento innovador en el ámbito universitario, demostrando un aumento de la motivación para aprender, potenciando el trabajo grupal, incentivando la búsqueda de información, aumentado la comprensión y la memorización del conocimiento. A su vez, el ABP pone énfasis en la interdisciplinariedad, aumenta el espíritu de colaboración del estudiante y su nivel de responsabilidad en el aprendizaje ${ }^{12}$.

Las nuevas orientaciones curriculares centran los objetivos en la adquisición de competencias profesionales. Este elemento competencial acredita el reconocimiento académico y permite la libre circulación de títulos universitarios. Partiendo de la Declaración de Bolonia de 1999, las universidades y los centros que imparten estudios superiores están llevando a cabo un proceso para promover la convergencia entre los sistemas nacionales de educación superior. Para ello se está desarrollando un Espacio Europeo de Educación Superior, que se implantará el año 2010, donde se evaluarán programas académicos convergentes que aseguren una calidad docente, adoptando el sistema de transferencia de créditos que permita un reconocimiento académico inmediato de títulos, una movilidad entre países y un aprendizaje a lo largo de toda la vida ${ }^{13}$. Según los parámetros de la convergencia europea educativa se debería considerar la introducción de un nuevo currículum basado en contenidos y competencias. Para ello se requeriría de una nueva definición de crédito universitario y considerarse éste como unidad de valoración de la actividad académica que integra armónicamente las enseñanzas teóricas y prácticas, otras actividades académicas dirigidas y el trabajo personal del estudiante, permitiendo medir el volumen total de trabajo que el estudiante debe realizar para superar cada una de las asignaturas ${ }^{14}$. El concepto de unidad de crédito debe reflejar el trabajo necesario para una formación académica integral. El aprendizaje debe desarrollar la capacidad de análisis del alumno aplicando lecciones magistrales, trabajos prácticos, seminarios, periodos de prácticas, trabajo de campo, trabajo personal - en bibliotecas o en el domicilio - así como los exámenes u otros posibles métodos de evaluación?

\section{ASUNCIÓN DE NUEVOS ROLES PARA EDUCADORES Y ESTUDIANTES}

Los requisitos que, tradicionalmente, se han exigido al profesor universitario, han cambiado en todos los estudios universitarios, pero se hacen más patentes en aquellas titulaciones en las que la formación está muy vinculada al ejercicio profesional. Es aconsejable fomentar un nuevo tipo de docentes, capaces de estimular y cultivar la independencia intelectual y la reflexión. Deberían ser conocedores de las TIC, adaptarse a los cambios sociales, conocer las novedades tecnológicas aplicadas a sus áreas de conocimiento, ser sensibles a las demandas de los diferentes sectores de producción y, por último, poseer las herramientas pedagógicas y docentes necesarias para transmitir y generar las actitudes, habilidades y conocimientos necesarios, posibilitando así la autosuficiencia profesional del alumnado.

El profesor debería convertirse en un elemento facilitador, en un guía y consejero sobre fuentes apropiadas de información, creador de hábitos y destrezas en la búsqueda, selección y tratamiento de la información ${ }^{15}$. Adquieren más importancia la experiencia como profesor y las habilidades de éste que la propia información. A la vez, el profesor debe utilizar los nuevos canales de la información para ayudar a los estudiantes en su autoformación.

Tres son las ideas ${ }^{16}$ que modifican las formas tradicionales de la enseñanza:

1. Enseñar a pensar, es decir, favorecer la curiosidad, la investigación, y la capacidad de crítica constructiva. A la vez, deberá enseñarse la tolerancia al error y la capacidad de extraer consecuencias positivas. Es conocido el concepto de "resiliencia" entendido como la capacidad de recuperar el estado de equilibrio después de un impacto negativo, cosa que se traduce en fortaleza, perseverancia y tenacidad ${ }^{17}$.

2. Enseñar a aprender, ofreciendo los medios para que una persona pueda disponer de la información de la metodología y contenidos necesarios, favoreciendo actitudes propensas al aprendizaje permanente.

3. Enseñar a ser creativos para tomar decisiones basadas en la intuición y la imaginación.

Por su parte, los estudiantes deben adoptar un papel mucho más importante en su formación, no sólo como elementos receptores, con una actitud pasiva, donde el único protagonismo consiste en asistir a clases magistrales o a prácticas de laboratorio de las que no entienden su utilidad, sino que deben participar de forma activa en la búsqueda, selección, procesamiento y asimilación de la información, en otras palabras deben sentir, experimentar y descubrir a través del aprendizaje significativo.

Los alumnos deben incorporar los nuevos conceptos por asimilación e incluirlos en los conocimientos previos. Asimismo, no deben estar muy alejados de su 
experiencia y de su realidad ya que si se alejan demasiado pueden llegar a resultar poco motivadores. Según Ausubel 18 existen cuatro tipos de aprendizaje:

1. Aprendizaje por recepción: el alumno recibe los contenidos en su forma final y éste sólo debe asimilar y comprender los contenidos.

2. Aprendizaje por descubrimiento: el alumno debe descubrir el contenido antes de asimilarlo.

3. Aprendizaje repetitivo: el alumno aprende los contenidos al pie de la letra.

4. Aprendizaje significativo: el alumno recibe el contenido de forma coherente, clara y organizada, el conocimiento es significativo y éste tiene una actitud favorable para aprender.

El proceso debería orientarse hacia el aprendizaje significativo, entendido como que se da a partir de la recepción i del descubrimiento personal. Los alumnos deben acceder a la información a través de la red, comunicándose con profesores expertos incluso de otras instituciones, intercambiando ideas y opiniones, accediendo a la información de diferentes publicaciones, congresos y simposios, etc. Para que el aprendizaje sea eficaz y duradero debe contemplar cinco características básicas:

1. Constructivo: proceso de construcción personal del conocimiento.

2. Activo: aprender para aplicar

3. Contextualizado: contexto espacio/tiempo determinado.

4. Social: tarea grupal (equipo de profesores y alumnos)

5. Reflexivo: conocer cómo aprendemos para superarnos. Respecto a este punto, cabe mencionar la frase de Savater ${ }^{19}$ en la que dice: "piense usted lo que quiera, pero piénselo"

\section{CARACTERÍSTICAS DEL APRENDIZAJE EN CIENCIAS DE LA SALUD}

En el ámbito de las Ciencias de la Salud, los métodos utilizados en el aprendizaje resultan instrumentos clave en el proceso formativo de personas competentes para el ejercicio de la profesión. Algunos autores $^{4,20}$ señalan la importancia del diseño curricular adaptado a las demandas percibidas por los alumnos, los recién graduados, los docentes, los profesionales en activo y la misma sociedad, poniendo énfasis en la integración de las diferentes necesidades formativas detectadas por los diferentes grupos. Ello permite prever errores, mejorar la calidad docente y, adaptar las necesidades de aprendizaje a las necesidades de salud de la población.
La diplomatura en Enfermería, así como muchas de las profesiones del ámbito sanitario, es una diplomatura con una vinculación muy directa sobre el ámbito laboral, puesto que la disciplina determina la capacidad y competencia de los graduados para poder ejercer en el ámbito del trabajo. En este sentido, el impacto de los cambios vividos por la sociedad (cambios económicos, demográficos, socioculturales, legales, políticos...) marca también nuevas directrices en los objetivos formativos de la profesión. Los cambios en las escalas de valores éticos, el choque de la sociedad de la información, el auge de nuevas asociaciones, las perspectivas de contratación laboral, el aumento de cultura en general por parte de la sociedad, son algunos de los elementos que cabe considerar para el diseño de los objetivos de aprendizaje. Este aspecto cobra importancia en la medida que el docente es el responsable de adaptar cuantas actualizaciones requiera la profesión a fin de que el alumno no tenga que "desaprender" a posteriori conceptos que ya no debieron haberse aprendido. El docente universitario, también debe hacer un esfuerzo por "desaprender", a fin de motivar un cambio y una evolución en su condición de docente. La capacidad de evolucionar no radica sólo en aprender muchas cosas, sino en la evolución que estos cambios generan, tanto a nivel personal como colectivo ${ }^{21}$. La metodología $\mathrm{ABP}^{22,23}$ resulta una buena estrategia de aprendizaje, puesto que consigue un incremento de la motivación por parte del alumno y de su grado de responsabilidad. La práctica del trabajo grupal en esta metodología resulta, a su vez, un valor añadido en el aprendizaje de la disciplina enfermera, puesto que responde positivamente a las exigencias requeridas en el ejercicio interdisciplinario profesional.

\section{CONCLUSIONES}

La Formación universitaria debe adaptarse a las exigencias profesionales de las diferentes disciplinas. La formación de profesionales con un alto nivel de capacitación adquiere una relevancia primordial para facilitar la inserción laboral de los recién graduados. La consecución de unos objetivos formativos que faciliten la capacitación de los estudiantes plantea una reflexión sobre los sistemas de aprendizaje que el estudiante utiliza. Los objetivos de aprendizaje deben orientarse a la consecución de la más alta competencia profesional. Para ello, el enfoque de aprendizaje debe centrarse en las diferentes estrategias que los estudiantes utilizan. Las TIC, el aprendizaje reflexivo, y la metodología $\mathrm{ABP}$ han resultado un incentivo para mejorar las condiciones de apren- 
dizaje. En el ámbito de Ciencias de la Salud, el aprendizaje mediante técnicas de grupo resulta un elemento facilitador para el desarrollo del ámbito profesional de la disciplina, aportando un valor añadido al conjunto de los conocimientos adquiridos. Estos cambios metodológicos suponen a su vez cambios paradigmáticos en la asunción de los roles de los estudiantes y, a su vez, del profesorado, el cual deberá adaptarse constantemente a las nuevas exigencias laborales, educativas y de aprendizaje.

Apostamos firmemente por este cambio conceptual en el proceso de aprendizaje. Sin embargo se plantean limitaciones en cuanto al beneficio que estas estrategias aportan al alumno puesto que existen muchos factores externos que pueden influir en el proceso y resultan difíciles de controlar. La competencia que estos alumnos puedan demostrar en el futuro ámbito laboral, evidenciará la efectividad de estas nuevas perspectivas formativas.

\section{BIBLIOGRAFÍA}

1. Bricall JM. Universidad 2000. Informe sobre la enseñanza superior en España. ed Madrid: conferencia de rectores de las universidades; 2000.

2. Gonzalez L. Hacia una educación pluridimensional. [en línea] noviembre 1997 [consulta: 7 de julio 2004] $<\mathrm{http} / / /$ www.crue.org/pgonzale.htm>

3. Oriol A. El coneixement i la competència professional. [en línea] 1997 [consulta: 06 de julio de 2004] < http://www.iesalut.es/pensaments/reflex_epist/reflex_e pist_index.asp>

4. Carreras J. Elementos de estrategia pedagógica e implicaciones metodológicas. Rev Educación médica 1999. Emero-marzo; vol 2, no 1:8-12.

5. Bologna Ministerial Meeting. The European Higher Education Area (better known as The Bolognia declaration).[documents a internet] Bologna: The National Unions of Students in Europe; junio de 1999 [citado el 11 de enero de 2005]. Disponible en:

http://www.esib.org/BPC/docs/Archives/CoP007_bologna_declaration.pdf

6. Bricall JM. La universidad, al final del milenio. [en línea] noviembre 1997.[consulta: 7 de julio 2004] <http://www.crue.org/pbricall.htm>

7. Asamblea de la CRUE. Declaración de Bologna: adaptación del sistema universitario español a sus directrices. [en línea] diciembre de 2000. [consulta: 15 de febrer 2002] <http://www.crue.org/apadsiuniv.htm>

8. Salinas J.Eneñanza flexible, aprendizaje abierto. Las redes como herramienta para la formación [en línea] Revista Electrónonica de Tecnologia Educativa, núm
10. Febrero 1999 [consulta: 7 de julio 2004]

<http://www.uib.es/depart/gte/revelec10.html>

9. Lewis R, Spencer D. What is Open Learning? CET, Open Learnin Guide 4. 1986

10. Holmberg B. Educación a distancia.Situación y perspectivas. Kapelusz. Buenos Aires.1985.

11. Topham P. The concept of "Openness" in relation to Computer Based Learning. Environments and management Education". Interactive Learning International. Vol.5 (1).198

12. Branda LA. El aprendizaje basado en problemas en la formación en Ciencias de la Salud. En :El aprendizaje basado en problemas: una herramienta para toda la vida. Escuela Universitaria de Enfermería de la Comunidad de Madrid; 2004 (en prensa)

13. Gonzalez J, Wagenaar R. Tuning educational structures in Europe. Informe final. Fase uno. Bilbao: Universidad de Deusto; 2003.

14. Agencia Nacional de Evaluación de la Calidad y Acreditación. "Programa de Convergencia Europea. El Crédito Europeo". Madrid; 2003

15. Adell J. Tendencias en educación en la sociedad de las tecnologías de la información. EDUTEC Revista Electrónica de Tecnologia Educativa, num. 7 Noviembre 1997

16. Renau E. Nuevas maneras de formar [en línea] Educaweb.com: 2000 [consulta: 7 de julio 2004] $<$ http://www.educaweb.com/esp/secciones/debate/text. asp?id=100321\&sec $=27 \&$ op $=4>$

17. Cedeño A. Aprender a emprender y fomentar el espíritu de iniciativa: una nueva tarea universitaria, Revista Acta Académica [en línea] noviembre 1999 (25) [consulta: 7 de julio 2004]

<http://www.uaca.ac.cr/acta/1999nov/acedeno.htm>

18. Ausubel DP. Psicología educativa: un punto de vista cognoscitivo. $2^{\mathrm{a}}$ ed. $2^{\mathrm{a}}$ reimpr. México: Editorial Trillas, 1987

19. Savater F. ¿De qué sirve la ética para los jóvenes? La educación es el momento adecuado de la ética. [en línea] Madrid Forum 1999. [consulta: 26 febrero 2002] $<$ http://www.terra.es/personal2/wwwally/otrasweb/ sabater.htm >

20. Manso JM. ¿Qué enseñar en Ciencias de la Salud? Técnicas para definir competencias y perfiles profesionales(y II) Rev. Educación Médica. Vol.3 n³, 2000

21. Dalmases M. La clave de aprender está en desaprender. Rev. Educación Médica. Vol 3, num.3, 2000

22. Mandin H, Jones A, Woloschuk W, Harasym P. Cómo ayudar a laos estudiantes a aprender a pensar como expertos cuando solucionan problemas clínicos. $\mathrm{Rv}$ Educación Médica. Vol 1 núm 2.:22-30.1998

23. Baños JE. El aprendizaje basado en problemas en los planes de estudio tradicionales: una alternativa posible. Rev Educación Médica.Vol 4, núm: 4-12. 2001. 